Article

\title{
Predictive Statistical Diagnosis to Determine the Probability of Survival in Adult Subjects with Traumatic Brain Injury ${ }^{\dagger}$
}

\author{
Mohammed Saleh ${ }^{1}$, Reza Saatchi ${ }^{1, *(\mathbb{D})}$, Fiona Lecky ${ }^{2}$ and Derek Burke ${ }^{3}$ \\ 1 Materials and Engineering Research Institute, Sheffield Hallam University, Sheffield S1 1WB, UK; \\ b3046810@my.shu.ac.uk \\ 2 Centre for Urgent and Emergency Care Research, Health Services Research Section, \\ School of Health and Related Research, University of Sheffield, Sheffield S10 2TH, UK; \\ f.e.lecky@sheffield.ac.uk \\ 3 Sheffield Children's Hospital, Western Bank, Sheffield S10 2TH, UK; Derek.Burke@sch.nhs.uk \\ * Correspondence: r.saatchi@shu.ac.uk \\ + This paper is an extended version of our paper published in AAATE2017 Congress Proceedings, \\ Sheffield, UK, 13-14 September 2017.
}

Received: 24 February 2018; Accepted: 4 April 2018; Published: 9 April 2018

\begin{abstract}
Determining the probability of survival after injury is important as it can inform triage, clinical research and audit. A number of methods have been reported for determining the probability of survival after injury. However, these have shortcomings and thus further developments are needed to improve their reliability and accuracy. In this study, a Bayesian method called Predictive Statistical Diagnosis (PSD) was developed to determine probability of survival in 4124 adults (age: mean $=67.9$ years, standard deviation $=21.6$ years) with traumatic brain injuries $(\mathrm{TBI})$. In total, $86.2 \%$ of cases had survived and $13.8 \%$ of cases had not survived their injuries. The parameters considered as inputs to PSD were age, abbreviated injury score (AIS), Glasgow coma score (GCS), pulse rate (PR), systolic blood pressure (SBP) and respiration rate (RR). PSD statistically modeled the TBI cases and their associated injury outcomes, i.e., survived or not survived. The model was calibrated on randomly selected, roughly $2 / 3$ (number 2676), of the cases and its performance was validated on the remaining cases (number 1448, i.e., validation dataset). The effectiveness of PSD in determining the probability of survival was compared with a method called Ps14 that uses regression modeling. With all parameters (i.e., age, AIS, GCS, SBP, RR and PR) included as inputs to PSD, it correctly identified $90.8 \%$ of survivors and $50.0 \%$ of non-survivors in the validation dataset while Ps14 identified $97.4 \%$ of survivors and $40.2 \%$ of non-survivors in the validation dataset. When age, AIS and GCS were used on their own as inputs to PSD, it correctly identified $82.4 \%$ of the survivors and $65.0 \%$ of non-survivors in the validation dataset. Age affected the performance of PSD in determining the survival outcomes. The number of non-surviving cases included in this study may have not been sufficiently high to indicate the full potential of PSD and a further study with a larger number of cases would be beneficial.
\end{abstract}

Keywords: traumatic brain injury; probability of survival; predictive statistical diagnosis; Bayesian modelling

\section{Introduction}

Injury is a significant cause of death and disability [1], accounting for $10 \%$ of global burden of deaths [2]. A number of injury severity scoring systems have been reported to quantify the severity of injury by considering measurable or observable status of the patient's medical condition [3]. Trauma 
scoring systems can be beneficial for [4,5]: (i) triage: a procedure to assess severity of medical condition for the purpose of setting treatment priority; (ii) prognostic evaluation: a procedure to support predication and management of injury outcomes and (iii) research: studies to compare patient groups on the basis of injury outcomes and assessing medical care and treatments. Trauma scoring systems can be classed as anatomical, physiological and combined. Anatomical scoring systems quantify the extent of individual anatomical injuries, taking into account the injury site by appropriate weightings (coefficients) however these weightings are often not known when the patient presents to hospital after a civilian trauma where most injury mechanisms are blunt (e.g., falls and road traffic collisions) [6]. Physiological scoring systems are based on cardiovascular, neurological and respiratory abnormalities. They provide mechanisms to determine the likelihood of mortality and inform triage; but can lack precision [6]. Combined anatomical and physiological scoring systems integrate the strengths of the anatomical and physiological scoring systems to improve their estimation of the probability of survival [7].

Trauma scores together with host factors such as sex, age and pre-existing medical condition are used in models to determine probability of survival [8-12]. The aim of this study is to investigate the effectiveness of a Bayesian method called Predictive Statistical Diagnosis (PSD) to determine probability of survival in Traumatic Brain Injury (TBI).

TBI can be defined as impaired brain function or other evidence of brain injury resulting from an external mechanical force, commonly a blunt force [13]. The injury mechanism could be classed as focal, diffuse and mixed [14,15]. Focal injuries result from blows to the head. These can result in laceration, contusion, and hemorrhage. Diffuse injuries are associated with stretching and twisting of axons and blood vessels by shear forces, resulting from acceleration, deceleration, and rotation of the brain. In mixed mechanisms both focal and diffuse mechanisms are present. Brain injuries resulting from incidents such as a blast could be mixed type. Brain injury can also be categorized as primary or secondary where primary injury is damage to the brain parenchyma as a result of the initial injury while the secondary injury occurs hours to days later as a result of the inflammatory effects that cause cerebral edema and cell depth [13]. TBI is the leading cause of death among those aged less than 45 years and in children aged between 1 to 15 years [15].

PSD was chosen as it is a robust statistical approach for developing models for differentiation between subject types. It has been successfully applied in studies for differentiating between healthy subjects and schizophrenic subjects based on a type of cognitive evoked potential extracted from the electroencephalogram (EEG) [16] and to identify an EEG extracted visual evoked potential component called the lambda wave [17]. In the following sections a brief explanation of current approaches to determine the probability of survival is provided, the concept of PSD is introduced, and our methodology and results are presented.

\section{Trauma Scoring Systems}

The Abbreviated Injury Scale (AIS) introduced in 1971 is a widely used anatomical injury severity scale [18]. AIS has been revised in 1980, 1985, 1990, 2005, 2008 and 2015. Its 1990 version (AIS-90) classifies more than 2000 injury types into nine body regions consisting of head, face, neck, abdomen, spine, upper extremities, lower extremities, and external [19]. An injury is given AIS scores in an ordinal scale from 1 (minor injury) to 6 (maximum injury, possibly lethal). In patients with multiple injuries, the maximum AIS score is used to describe the overall severity but this has been reported to not correlate linearly with probability of death [4]. A derivative of AIS called Injury Severity Score (ISS) [20] was introduced to facilitate combining anatomical injuries from multiple sites. The ISS has a range of 1 to 75 and is calculated by grouping the nine AIS body regions into six (head or neck, face, chest, abdominal or pelvic contents, extremities or pelvic girdle, and external) and then summing the squares of the highest AIS values for three most severely injured body regions [21].

One of the most consistent features of TBI-impairment of brain function or other evidence of brain damage resulting from an external force is impairment of consciousness [22-24]. Glasgow Coma Scale (GCS) was introduced by Jennett and Teasdale (1977) [24] to assess the level of consciousness 
in a wide range of disorders and thus it has a role in grading TBI [25,26]. In the GCS, assessment is performed by examining the extent of eye opening, verbal response and motor response as indicated in Table 1.

Table 1. Glasgow Coma Scale (GCS) components.

\begin{tabular}{ccc}
\hline Best Motor Response (M Score) & Best Verbal Response (V Score) & Eye Opening (E Score) \\
\hline Moves limb to command (6) & Oriented (5) & Spontaneous (4) \\
Localizes to painful stimulus (5) & Confused (4) & Open to speech (3) \\
Withdraws from painful stimulus (4) & Inappropriate words (3) & Open to pain (2) \\
Abnormal flexion response (3) & Incomprehensible words (2) & None (1) \\
Abnormal extension response (2) & No verbal (1) & - \\
No motor response (1) & & - \\
\hline
\end{tabular}

The GCS is the sum of its three components, i.e., eye opening score + verbal score + motor score. The eye-opening component examines the arousal mechanism of the brainstem; the verbal component examines the integration of cerebral cortex and brainstem and the motor response examines the integrity of cerebral cortex and spinal cord [27].

TBI can be classed as mild, moderate or severe. TBI with GCS of 13 to 15 is graded as mild. TBI with GCS of 9 to 12 is graded as moderate and is associated with prolong loss of consciousness, abnormal neuroimaging, and neurological deficit. Patients in this category may need neurological evaluation and intervention [26]. GCS of 8 or less is graded as severe TBI. These patients have significant neurological injury and typically have abnormal neuroimaging observed from Computed Tomography (CT) such as a skull fracture, traumatic intracranial hemorrhage, or cerebral contusion $[26,28]$.

Although GCS is valuable for TBI assessment, it has some limitations. For example brain stem reflex eye movements are not considered [29]. Champion et al. (1989) [30] introduced a physiological trauma assessment system called Revised Trauma Score (RTS) that incorporates the GCS, systolic blood pressure and respiratory rate as shown in Table 2 . This index is determined by adding up the results from the values of the three components and multiplying them by their corresponding weights.

Table 2. Revised Trauma Score [30].

\begin{tabular}{cccc}
\hline Code & Glasgow Coma Scale & Systolic Blood Pressure (mmHg) & Respiratory Rate (Breaths Per Minute) \\
\hline 4 & $13-15$ & $>89$ & $10-29$ \\
3 & $9-12$ & $76-89$ & $>29$ \\
2 & $6-8$ & $50-75$ & $6-9$ \\
1 & $4-5$ & $1-49$ & $1-5$ \\
0 & 3 & - & - \\
\hline
\end{tabular}

\section{Approaches to Determine Probability of Survival}

Trauma and Injury Severity Score (TRISS) is a method that combines anatomical and physiological scoring systems to determine the probability of trauma survival $\left(p_{s}\right)$ for adults sustaining traumatic injuries from blunt and penetrating mechanisms [31]. The probability of survival using this system is given by

$$
\begin{gathered}
p_{S}=\frac{1}{1+e^{-b}} \\
b=\alpha_{i}+\beta_{A G E, i} \times \mathrm{AGE}+\beta_{R T S, i} \times \mathrm{RTS}+\beta_{I S S, i} \times \mathrm{ISS}
\end{gathered}
$$

where $i=1$ is for blunt mechanism and $i=2$ is for penetrating mechanism, $\alpha_{i}$ is a constant for mechanism $i, \beta_{A G E, i}$ is the coefficient related to age and mechanism $i, \beta_{R T S, i}$ is the coefficient associated with RTS and mechanism $i, \beta_{I S S, i}$ is the coefficient associated with ISS and mechanism $i$. RTS is obtained by 


$$
\mathrm{RTS}=\beta_{R R} \times \mathrm{RR}+\beta_{S B P} \times \mathrm{SBP}+\beta_{G C S} \times \mathrm{GCS}
$$

where $\beta_{R R}$ is the coefficient associated with respiration rate (RR), $\beta_{S B P}$ is the coefficient associated with systolic blood pressure (SBP), and $\beta_{G C S}$ is the coefficient associated with GCS. TRISS however has a number of shortcomings related to calibration of its coefficients, variable inter-relationships or interactions and strong linear assumptions between predictor variable and survival outcome [31,32]. Adjustments to its coefficients result in performance variations by TRISS in predicting probability of survival for trauma patients [33].

In 2004, the Trauma Audit and Research Network (TARN) [34] proposed a Probability of Survival model called Ps04. This model uses age, gender, their interaction, ISS and GCS (including a variable for intubation). In 2014, TARN introduced the Ps14 model by incorporating the Charlson Comorbidity Index (CCI) [35] to the assess Pre-Existing Medical Conditions (PMC). To predict probability of survival in Ps14, Ps04 parameters and CCI are required. It determines the percentage of probability of survival by performing retrospective measure of a new patient by comparison with those of a similar profile on the TARN database. For example, if $p_{s}=53 \%$, then on average 53 out of every 100 patients have survived and 47 patients have not survived.

$$
p_{s}=\frac{e^{b}}{1+e^{-b}}
$$

where $e=2.718282$ and $b$ is defined as the linear combination of the regression coefficients and the values of the corresponding patient's characteristics (ISS, GCS, modified CCI, age and gender).

\section{Predictive Statistical Diagnosis}

Predictive statistical diagnosis (PSD) uses Bayesian statistics to determine to which of a given set of predefined types $t$, a measurement expressed by a feature vector $(x)$ belongs [36,37]. It uses example cases of known types, represented in a training data set to obtain the values of its calibration parameters. Once these parameters are calibrated, it can classify an unknown case into the types represented by $t$.

The probability that an observation vector $x$ and parameter vector $\theta$ belongs to the type $t_{1}$ is given by Bayesian statistics as

$$
p\left(t_{1} \mid x, \boldsymbol{\theta}\right)=\frac{p\left(t_{1}\right) p\left(\boldsymbol{x} \mid t_{1}, \boldsymbol{\theta}\right)}{p(\boldsymbol{x})}
$$

where $p\left(t_{1}\right)$ is the prior probability of type $t_{1}, p\left(x \mid t_{1}, \boldsymbol{\theta}\right)$ is the probability density function of $x$ for a given type $t_{1}$. Equation (4) can be rewritten as predictive density function for an observation $x$ on a case of type $t$ assessed on the training data $Z$ as $[36,37]$.

$$
p\left(t_{1} \mid \boldsymbol{x}, \boldsymbol{\theta}\right)=\frac{p\left(t_{1}\right) q\left(\boldsymbol{x} \mid t_{1}, \boldsymbol{Z}\right)}{\sum_{t=t_{1}}^{t_{n}} p(t) q(\boldsymbol{x} \mid t, \boldsymbol{Z})}
$$

To determine above, $q\left(x \mid t_{1}, Z\right)$ can be replaced with [37]

$$
q\left(\boldsymbol{x} \mid t_{1}, \mathbf{Z}\right)=S t_{d}\left(v_{t}, \boldsymbol{m}_{t},\left(1+\frac{1}{n_{t}}\right) \boldsymbol{S}_{t}\right)
$$

where there are $n_{t}$ cases of type $t$ with feature vectors $x_{1}, x_{2}, \ldots x_{n} ; v_{t}$ is the degrees of freedom given by $n_{t}-1, m_{t}$ and $S_{t}$ are the mean and the covariance matrices respectively. $S t_{d}$ represents a $d$-dimensional student $t$ density determined as 


$$
S t_{d}(v, \boldsymbol{b}, \boldsymbol{c})=\frac{\Gamma[0.5(v+1)]}{\pi^{0.5 d} \Gamma\{[0.5(v-d+1)]\}|v \boldsymbol{c}|^{0.5}} \times \frac{1}{\left[1+(\boldsymbol{x}-\boldsymbol{b})^{T}(v \boldsymbol{c})^{-1}(\boldsymbol{x}-\boldsymbol{b})^{0.5(v+1)}\right]}
$$

where the variables $v, b$ and $c$ relate to Equation (6) as $v=v_{t}, b=m_{t}$ and $c=\left(1+\frac{1}{n_{t}}\right) \boldsymbol{S}_{t}$. $\Gamma$ is the gamma function, $T$ and -1 represent matrix transpose and inversion operations, respectively. Using Equation (5), $p\left(t_{1} \mid \boldsymbol{x}, \boldsymbol{\theta}\right)$ is determined for the cases of known types. Then to compute the probabilities for the unknown cases (i.e., those on the validation data set), Equation (7) uses the observation vector $x$ for cases of known types but retains the mean $\left(\boldsymbol{m}_{t}\right)$ and covariance $\left(S_{t}\right)$ matrices to identify an unknown type. The parameters $m_{t}$ and $S_{t}$ are calibration information for the PSD.

\section{Methodology}

The TBI data used in this study were provided by the TARN [34]. They provided a subset of their very large database excluding the cases that were not relevant to this study. Only adults with TBI that had full record of age, GCS, AIS, respiration rate, pulse rate, systolic blood pressure and their associated Ps14 were selected for this analysis. Subjects aged less than 17 years were excluded as their injury survival characteristics were considered to be different from the adults and thus their inclusion with adults in a single model would have made the model more complex. The number of available cases aged less than 17 with TBI was considered insufficient for developing such a model. Trauma deaths for subjects who were less than 17 years old in the U.K. (where this study was carried out) is rare relative to the adults. Children in the TARN database were treated in different wards and hospitals to the adults. Furthermore, most organ systems are mature by 12 years, while bones can grow until age 21 years. However, this does not impact on trauma mortality. A statistical summary indicating sex, age and number of the cases included in the study is provided in Table 3.

Table 3. Information summary for adult TBI cases (total 4124) included in the study.

\begin{tabular}{cccccc}
\hline \multicolumn{2}{c}{ Gender } & \multicolumn{2}{c}{ Age (Years) } & \multicolumn{2}{c}{ Injury Outcomes } \\
\hline Male & Female & Mean & Standard Deviation & Survived & Did Not Survive \\
\hline $2488(60.3 \%)$ & $1636(39.7 \%)$ & 67.9 & 21.6 & $3553(86.2 \%)$ & $571(13.8 \%)$ \\
\hline
\end{tabular}

Calibration (training) and validation data sets were created. The calibration data set contained roughly $2 / 3$ of the cases (number $=2676$ ) and was used to calibrate the PSD. The validation data set contained the remaining subjects (number $=1448$ ) and was used to determine PSD's performance on cases not included in the calibration set. The decision to use roughly 2/3 of the cases for calibration and the rest for validation was based on including representative injury patterns for performing PSD calibration and to validate it on the remaining cases. Adult subjects whose full set of parameters for input to PSD were unavailable were excluded.

Figure 1 shows age information plots for the subjects. Figure 1a shows the age boxplots for the subjects that survived and those that did not survive. The median of age ( 83.7 years) for subjects that did not survive is higher than for the subjects that survived (71.6 years). Figure $1 \mathrm{~b}$ shows the age distribution of all subjects. Figure $1 \mathrm{c}, \mathrm{d}$ show the age distributions for subjects that survived and those that did not survive respectively. The subjects in calibration and validation datasets were randomly selected from the complete set through a random number generator function. Therefore, the cases in each set had comparable age, AIS, GCS, respiration rate, systolic blood pressure and pulse rate values.

From here onward in the paper, the results relate to the subjects included in the validation dataset only as these subjects are basis for analyzing the performance of PSD.

Figure $2 \mathrm{a}, \mathrm{b}$ show the age distributions of the subjects that are included in the validation set separated into those that survived (Figure 2a) and those that did not (Figure 2b). 


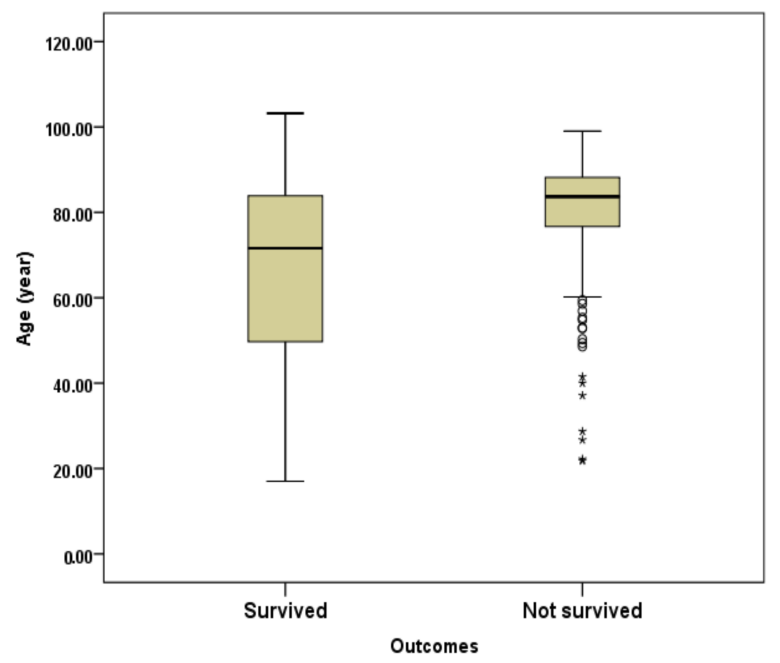

(a)

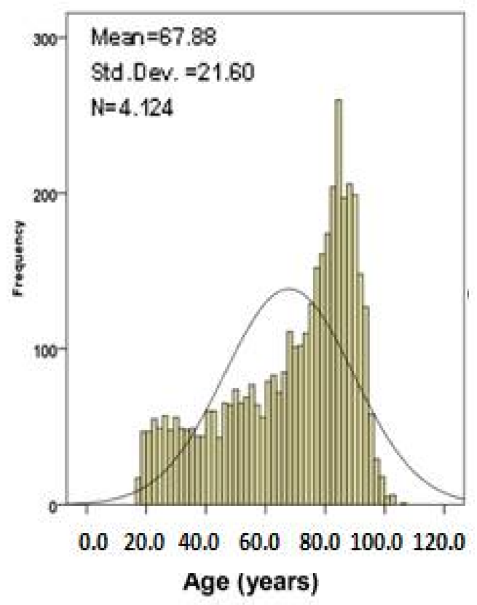

(b)

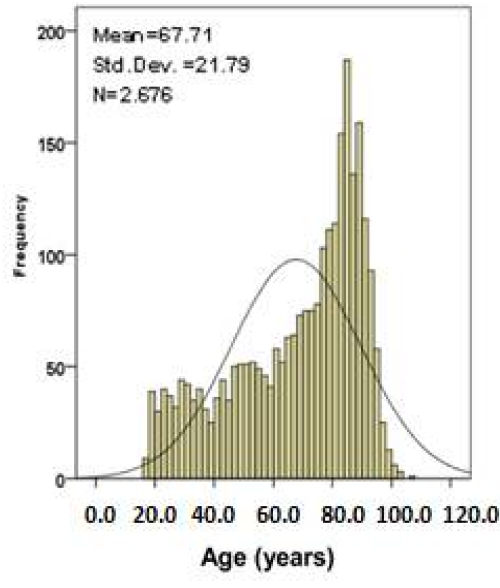

(c)

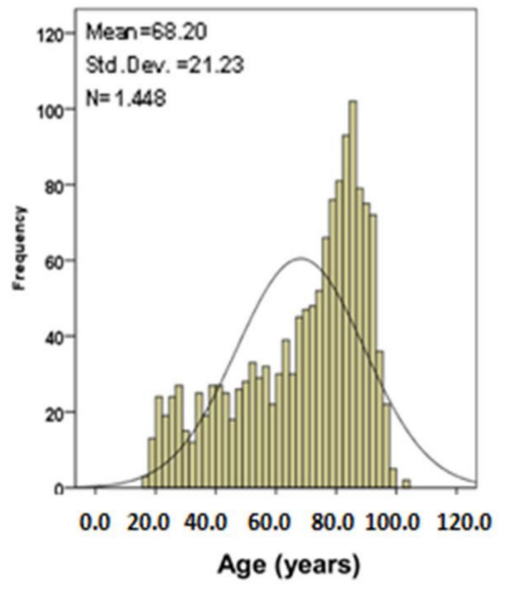

(d)

Figure 1. (a) Age boxplots for the subjects that survived and those that did not survive; (b) age distribution for all subjects; (c) age subjects of subjects in calibration set; (d) age distribution of subjects in validation set.

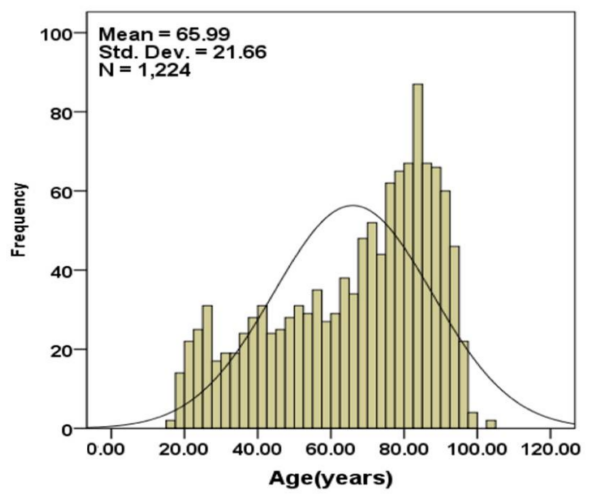

(a)

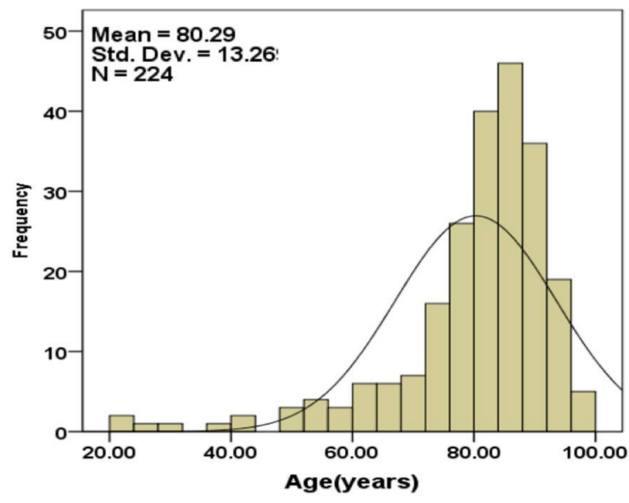

(b)

Figure 2. (a) Age distributions of the subjects in the validation set for the cases that (a) survived and (b) had not survived.

The age statistics for subjects included in the validation set is summarized in Table 4 . 
Table 4. Age (in years) statistical summary for subjects in the validation dataset.

\begin{tabular}{cccc}
\hline \multirow{2}{*}{ Parameter } & All Subjects & Survived & Did Not Survive \\
\cline { 2 - 4 } & $\mathbf{1 4 4 8}$ & $\mathbf{1 2 2 4}$ & $\mathbf{2 2 4}$ \\
\hline Mean & 68.2 & 66.0 & 80.3 \\
Median & 75.1 & 71.6 & 83.7 \\
Mode & 87.5 & 87.5 & 85.7 \\
Standard deviation & 21.2 & 21.7 & 13.6 \\
Variance & 450.7 & 469.5 & 176.1 \\
Range & 86.2 & 86.2 & 77.2 \\
Minimum & 17.0 & 17.0 & 21.8 \\
Maximum & 103.2 & 103.2 & 99.0 \\
\hline
\end{tabular}

The relationships between age, sex, AIS and GCS for the cases included in the validation data sets are shown separately in Figure 3 for the cases that survived and in Figure 4 for cases that did not survive. There is a large cluster of surviving cases associated with AIS $=4$ and 5 and GCS $=15$ (mild injury severity). The subjects that did not survive are primarily clustered at AIS $=5$ and tend to be more than 70 years. The relationship between sex (male/female) and survival outcomes from Figures 3 and 4 should be treated with caution as the complete set contained 1.521 times more males than females (i.e., 2488 male cases and 1636 female cases).

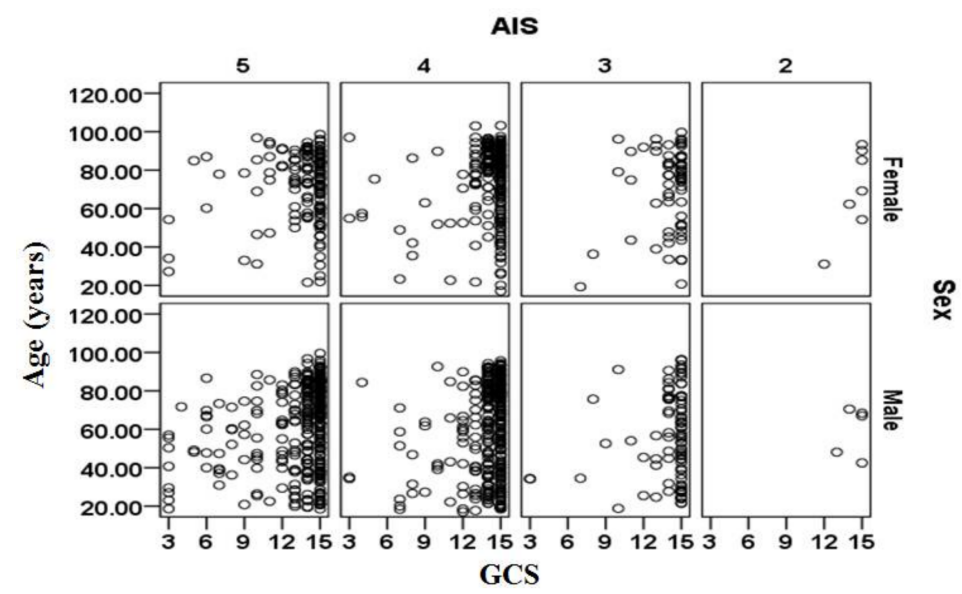

Figure 3. Relationships between age, sex, abbreviated injury score (AIS) and Glasgow coma score (GCS) for the subjects included in the validation dataset that survived.

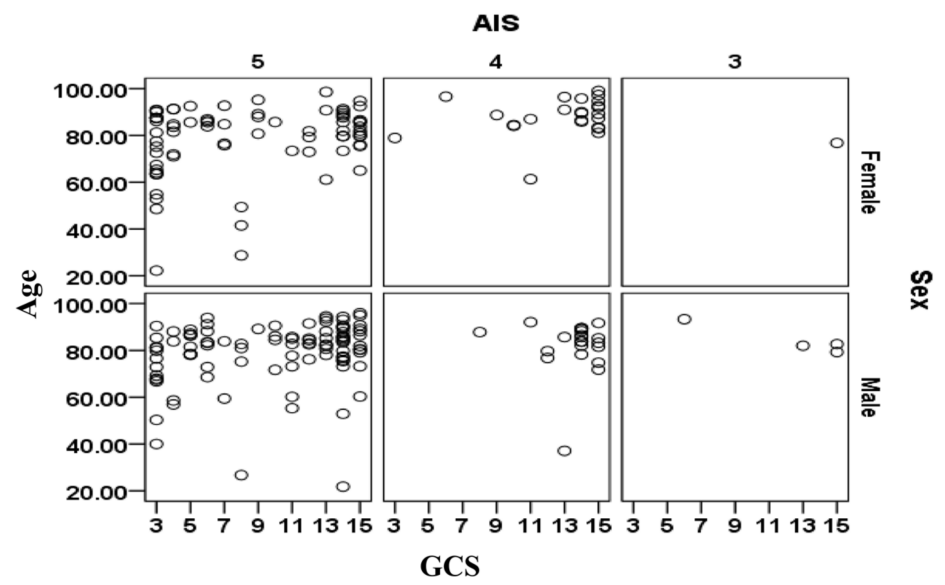

Figure 4. Relationships between age, sex, AIS and GCS for the subjects included in the validation dataset that had not survived. 
The values of the Ps14 were already available from the TARN database and so we only used these existing values for comparison with PSD. The parameters considered as input to PSD were AIS, GCS, respiratory rate, pulse (heart) rate, systolic blood pressure (SBP), sex (male/female) and age. In order to be able to analyze these parameters more effectively, their values (except AIS) were categorized as shown in Table 5 [38]. The categorization allocated the values of these variables into predefined groupings, i.e., normal, abnormal mild, moderate severe. The advantages of this operation are that the inter-class variations within the measured variables could be reduced and the results could be interpreted more specifically into severity types. The disadvantage this categorization is, however, that actual readings are replaced by their category types.

Table 5. Categorization of Glasgow coma score (GCS), pulse rate (PR, beats per minute, bpm), respiratory rate (RR, breaths per minute, bpm) and systolic blood pressure.

\begin{tabular}{cccc}
\hline Measures & Range & Category \\
\hline \multirow{2}{*}{ GCS } & Score 13-15 & 3 (Mild) \\
& Score 9-12 & 2 (Moderate) \\
& Score 3-8 & \multicolumn{2}{c}{1 (Severe) } \\
\hline Pulse rate & $60-100 \mathrm{bpm}$ & Normal $=2$ & Abnormal $=1$ \\
Respiratory rate & $14-20 \mathrm{bpm}$ & Normal $=2$ & Abnormal $=1$ \\
Systolic blood pressure & $90-140 \mathrm{mmHg}$ & Normal $=2$ & Abnormal $=1$ \\
\hline
\end{tabular}

PSD required the prior probability for not surviving to be specified as part of its operation (prior probability of survival $=1$-prior probability for not survival). To determine the most suitable value for this prior probability, prior probability values between 0 and 1 were experimented and for each value the percentage correct identifications for the survived and did not survive cases for the calibration (training) dataset were obtained. Figure 5 shows the plot of these results. The graphs indicated that highest identification accuracy was for prior probability equal to 0.27 and this value was chosen for the rest of the analysis (only a section centered on 0.27 is shown in the figure).

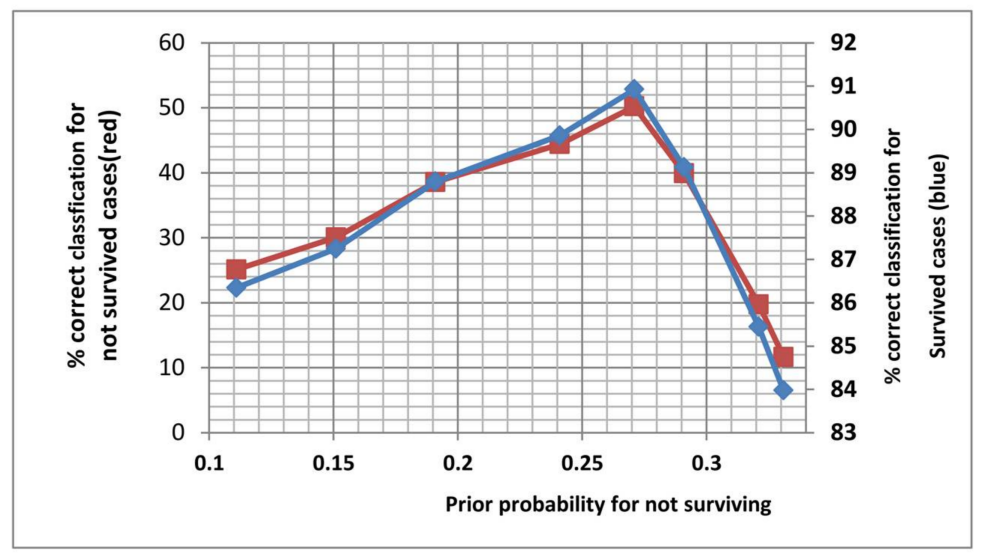

Figure 5. The relationship between the prior probability of not surviving and the associated percentage correct identification for the survived (blue plot) and not survived (red plot) cases. The subjects were from the calibration set. Only the section centered on the peak (i.e., prior probability $=0.27$ ) is shown in this figure.

\section{Results and Discussion}

Figure 6 shows the interrelationships between pulse rate, systolic blood pressure, GCS, AIS, and respiration rate information for non-surviving cases included in the validation set. A large cluster of cases appears for AIS $=5$, GCS $=1$ (categorized as severe injury) and systolic pressure $=1$ (categorized abnormal). 


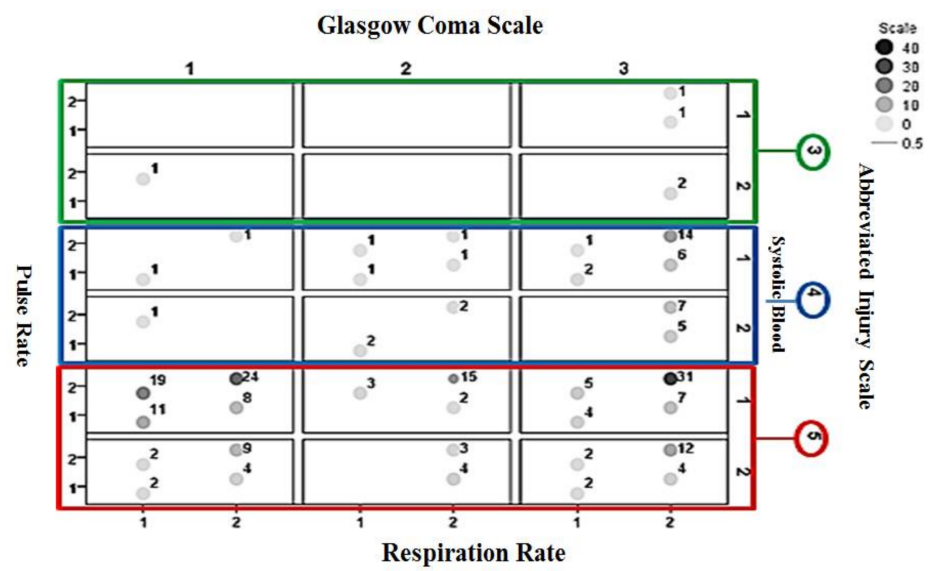

Figure 6. The interrelationships between injury parameters for non-surviving cases. The values next to the circles indicate the number of associated cases. Larger values are highlighted by darker circles. Subjects are from the validation dataset.

Figure 7 shows the identification results using Ps14 for non-surviving cases included in the validation dataset. Figure $7 \mathrm{a}$ is for those correctly identified and Figure $7 \mathrm{~b}$ is for cases misidentified. A larger proportion of correctly identified cases are associated with AIS $=5$, GCS $=1$ (categorized as severe injury) and a large proportion of misidentified cases are associated with AIS $=5$, GCS $=3$ (categorized as mild injury).

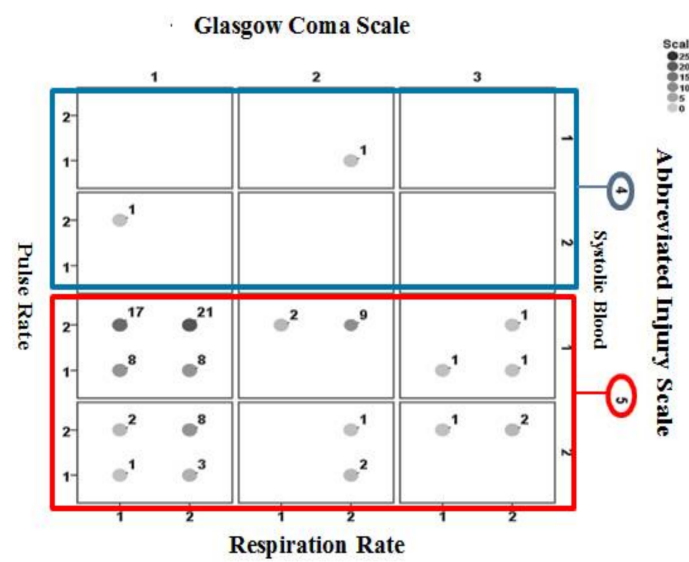

(a)

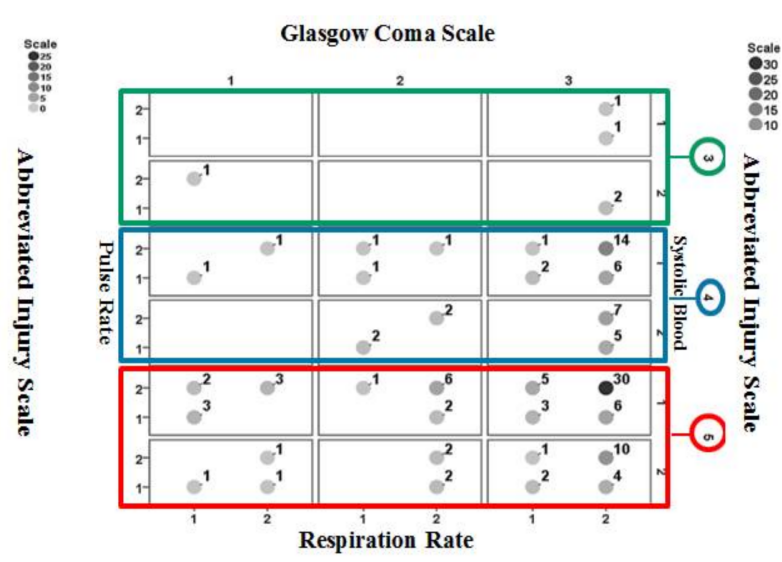

(b)

Figure 7. Identification results for Ps14 for non-surviving cases in the validation dataset: (a) correctly identified cases (b) misidentified cases. The values next to the circles indicate the number of associated cases.

Figure 8 shows the identification results obtained using PSD for non-surviving cases included in the validation dataset. Figure $8 \mathrm{a}$ is for those correctly identified and Figure $8 \mathrm{~b}$ is for those misidentified. Results consistent to those from Ps14 are observed where a larger proportion of correctly identified cases are associated with AIS $=5$, GCS $=1$ (categorized as severe injury) and a large proportion of misidentified cases are associated with AIS $=5, \mathrm{GCS}=3$ (categorized as mild injury).

Table 6 provides an analysis of injury patterns and performance of PSD and Ps14 in identifying non-surviving cases included validation dataset. An X in the last 5 columns of the table indicates the associated parameter is categorized as abnormal, serious injury (for AIS 3 to 5) or as severe injury (for GCS). The table shows that in some injury patterns Ps14 has performed better than PSD and vice versus. For example, the injury pattern resulting with the largest number of non-surviving cases (i.e., 31 cases, expressed as $\mathrm{X}_{22153}$ ) is associated with pulse rate $=2$ (categorized as normal category), 
respiration rate $=2$ (categorized as normal category), systolic blood pressure $=1$ (categorized as abnormal category), AIS $=5$ (critical) and GCS $=3$ (categorized as mild injury). Only one of the associated cases has been correctly identified by Ps14 however 6 were correctly identified by PSD. There were 24 cases associated with the injury pattern $X_{22151}$. For this injury pattern pulse rate $=2$ (categorized as normal category), respiration rate $=2$ (categorized as normal category), systolic blood pressure $=1$ (categorized as abnormal category), AIS $=5$ (critical) and GCS $=1$ (categorized as severe injury). Ps14 has performed better than PSD by correctly identifying from 21 out of 24 cases while PSD identified 18 cases correctly. For some injury patterns the identification accuracy of the two models (PSD and Ps14) is $0 \%$. An example for this is injury pattern $\mathrm{X}_{22143}$. This associates with pattern pulse rate $=2$ (categorized as normal category), respiration rate $=2$ (categorized as normal category), systolic blood pressure $=1$ (categorized as abnormal category), AIS $=4$, and GCS $=3$ (categorized as mild injury). The reason why PSD and Ps14 performance differ or in some injury patterns they fail to identify the outcome correctly requires further investigation.

Table 7 compares the results obtained using PSD and Ps14 to determine the probability of survival in cases included in the validation dataset. The inputs to PSD were AIS, GCS, age, systolic blood pressure, respiration rate and pulse rate. Ps14 correctly identified $97.4 \%$ of surviving cases and $40.2 \%$ of the non-surviving cases. However PSD correctly identified $90.8 \%$ of the surviving cases and $50.0 \%$ of the non-surviving cases. These results indicate the main difference between the two methods relates to their abilities to identify the non-survivors.

Figure $9 \mathrm{a}, \mathrm{b}$ provide a further analysis of the results in Table 7 . The figures indicate the number of surviving and non-surviving cases correctly identified by Ps14 and PSD and the overlap in the number of cases correctly identified by both methods.

The results in Table 7 are taken further by considering the effect of age on the performance of PSD and Ps14. The cases included in the validation that did not survive were divided into two groups (i) those aged between 17 and 65 years and (ii) those aged above 65 years. Age 65 was considered as the boundary as criteria for immediate CT scan of the head in adults with traumatic brain injury include age more than 65 years and some loss of consciousness or amnesia since the injury [39]. In the Canadian CT Head-Rules traumatic head injury patients aged 65 are classed as high risk that warrant a CT of the head [40]. The results obtained are shown in Table 8. PSD has higher identification accuracy for both age groups as compared with Ps14. Comparing the identification results for the two age groups; the performance of both models is influenced by the considered age ranges. Ps14 has been more accurate for cases aged above 65 years than those between 16-65 years. PSD on the other hand has been much more accurate for cases aged 17-65 years as compared with those aged over 65 years.

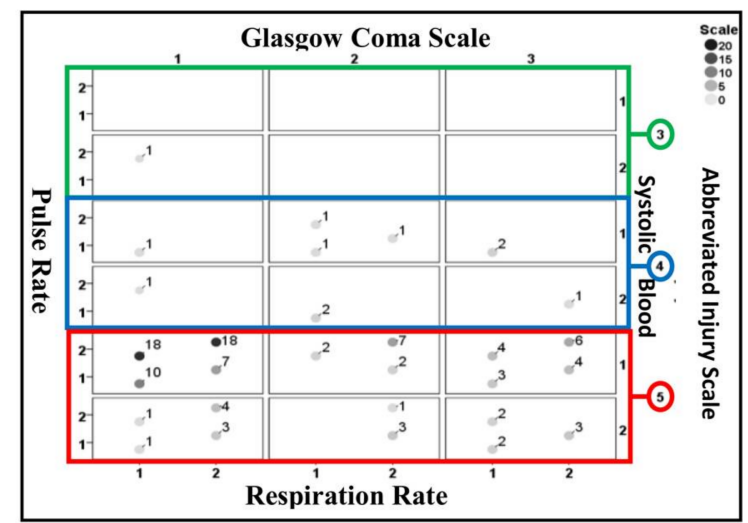

(a)

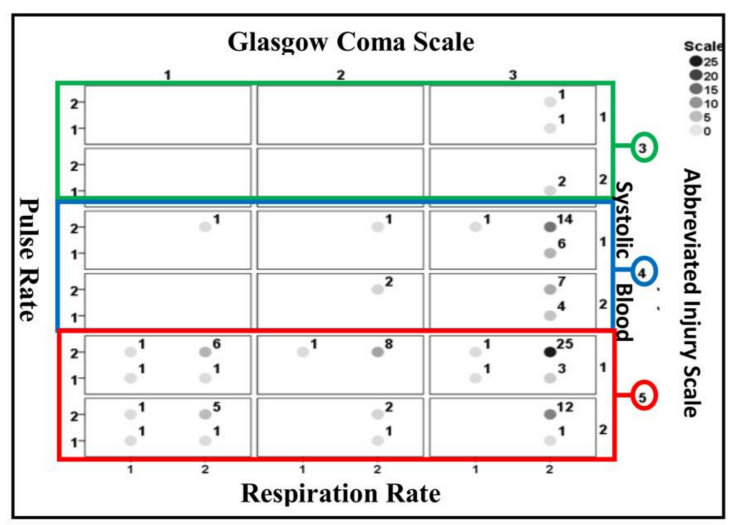

(b)

Figure 8. Identification results for PSD for non-surviving cases included in the validation dataset. (a) Correctly identified cases (b) misidentified cases. The values next to the circles indicate the number of associated cases. 
Table 6. Analysis of injury patterns for non-surviving cases included in the validation dataset (the patterns with relatively small number of cases are not shown). An $\mathrm{x}$ in the trauma parameter columns indicates abnormal or severe categorization for the related parameter.

\begin{tabular}{|c|c|c|c|c|c|c|c|c|}
\hline \multirow{2}{*}{ Injury Scenarios } & \multirow{2}{*}{$\begin{array}{c}\text { Number of Cases That } \\
\text { Did Not Survive (Figure 6) }\end{array}$} & \multirow{2}{*}{$\begin{array}{l}\text { Number of Cases Correctly } \\
\text { Identified by Ps14 (Figure 7a) }\end{array}$} & \multirow{2}{*}{$\begin{array}{l}\text { Number of Cases Correctly } \\
\text { Identified by PSD (Figure 8a) }\end{array}$} & \multicolumn{5}{|c|}{ Trauma Parameter } \\
\hline & & & & AIS & GCS & SBP & RR & PR \\
\hline$X_{22153}$ & 31 & $1(3.2 \%)$ & $6(19.4 \%)$ & $x$ & & $x$ & & \\
\hline $\mathrm{X}_{22151}$ & 24 & $21(87.5 \%)$ & $18(75.0 \%)$ & $\mathrm{x}$ & $\mathrm{x}$ & $\mathrm{x}$ & & \\
\hline $\mathrm{X}_{21151}$ & 19 & $17(89.5 \%)$ & $18(94.7 \%)$ & $\mathrm{x}$ & $\mathrm{x}$ & $\mathrm{x}$ & & \\
\hline$X_{22152}$ & 15 & $9(60.0 \%)$ & $7(46.7 \%)$ & $\mathrm{x}$ & $\mathrm{x}$ & $\mathrm{x}$ & & \\
\hline $\mathrm{X}_{22143}$ & 14 & $0(0.0 \%)$ & $0(0.0 \%)$ & $\mathrm{x}$ & & $\mathrm{x}$ & & \\
\hline $\mathrm{X}_{22253}$ & 12 & $2(16.7 \%)$ & $0(0.0 \%)$ & $\mathrm{x}$ & & & & \\
\hline $\mathrm{X}_{11151}$ & 11 & $8(72.7 \%)$ & $10(90.9 \%)$ & $\mathrm{x}$ & $\mathrm{x}$ & $\mathrm{x}$ & $\mathrm{x}$ & $\mathrm{x}$ \\
\hline $\mathrm{X}_{22251}$ & 9 & $8(88.9 \%)$ & $4(44.4 \%)$ & $\mathrm{x}$ & & & & $\mathrm{x}$ \\
\hline $\mathrm{X}_{12151}$ & 8 & $8(100.0 \%)$ & $7(87.5 \%)$ & $\mathrm{x}$ & $\mathrm{x}$ & $\mathrm{x}$ & & \\
\hline $\mathrm{X}_{21243}$ & 7 & $0(0.0 \%)$ & $0(0.0 \%)$ & $\mathrm{x}$ & & & & $\mathrm{x}$ \\
\hline $\mathrm{X}_{12153}$ & 7 & $1(14.3 \%)$ & $4(57.1 \%)$ & $\mathrm{x}$ & & $\mathrm{x}$ & & $\mathrm{x}$ \\
\hline $\mathrm{X}_{12143}$ & 6 & $0(0.0 \%)$ & $0(0.0 \%)$ & $x$ & & $\mathrm{x}$ & & $\mathrm{x}$ \\
\hline $\mathrm{X}_{12243}$ & 5 & $0(0.0 \%)$ & $1(20.0 \%)$ & $\mathrm{x}$ & & & & $\mathrm{x}$ \\
\hline$X_{21153}$ & 5 & $0(0.0 \%)$ & $4(80.0 \%)$ & $\mathrm{x}$ & & $\mathrm{x}$ & $\mathrm{x}$ & \\
\hline $\mathrm{X}_{11153}$ & 4 & $1(25.0 \%)$ & $3(75.0 \%)$ & $\mathrm{x}$ & & $\mathrm{x}$ & $\mathrm{x}$ & $\mathrm{x}$ \\
\hline $\mathrm{X}_{12251}$ & 4 & $3(75.0 \%)$ & $3(75.0 \%)$ & $\mathrm{x}$ & $\mathrm{x}$ & & & $\mathrm{x}$ \\
\hline $\mathrm{X}_{12252}$ & 4 & $2(50.0 \%)$ & $3(75.0 \%)$ & $\mathrm{x}$ & $\mathrm{x}$ & & & $\mathrm{x}$ \\
\hline $\mathrm{X}_{12253}$ & 4 & $0(0.0 \%)$ & $3(75.0 \%)$ & $\mathrm{x}$ & & & & $\mathrm{x}$ \\
\hline
\end{tabular}

$\mathrm{X}_{\text {abcde }}$ : The subscript "a" represents pulse rate (categorized as 1 abnormal, 2 normal), " $\mathrm{b}$ " represents respiration rate (categorized as 1 abnormal, 2 normal), "c" represents systolic blood pressure (categorized as 1 abnormal, 2 normal), " $\mathrm{d}$ " represents AIS and " $\mathrm{e}$ " represents GCS (1: severe, 2: moderate and 3: mild).

Table 7. Comparison of PSD and Ps14 to predict probability of survival for cases in the validation dataset (when probability value was greater than or equal to 0.5 , the subject was classed as surviving and when probability value was less than 0.5 , the subject was classed as not surviving).

\begin{tabular}{cccccc}
\hline \multicolumn{2}{c}{ Number of Cases } & \multicolumn{2}{c}{ Ps14 } & \multicolumn{2}{c}{ PSD } \\
\hline Survived & Did Not Survive & Survived & Did Not Survive & Survived & Did Not Survive \\
\hline 1224 & 224 & $1192(97.4 \%)$ & $90(40.2 \%)$ & $1112(90.8 \%)$ & $112(50.0 \%)$ \\
\hline
\end{tabular}

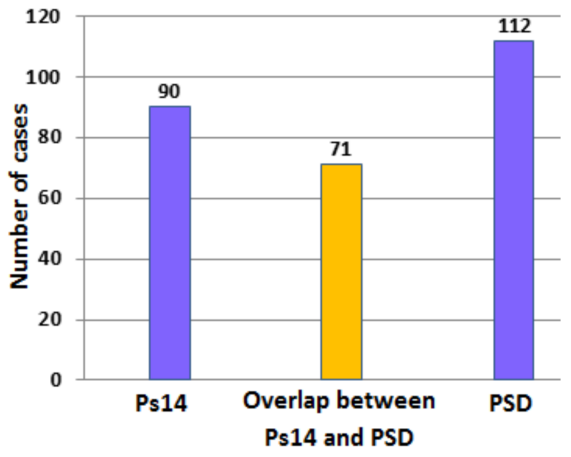

(a)

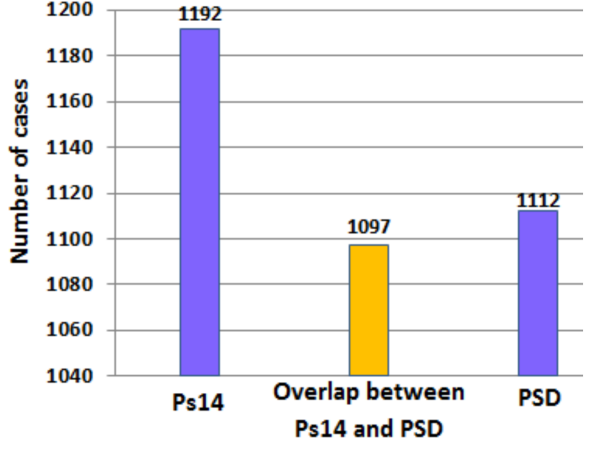

(b)

Figure 9. The number of cases in the validation set correctly identified by Ps14 and PSD (a) non-surviving cases; (b) surviving cases. The middle bar indicates the overlap in correct identification of cases by both Ps14 and PSD.

Table 8. Performance comparison of PSD and Ps14 based on age groups for cases in the validation dataset that had not survived.

\begin{tabular}{cccccccccc}
\hline $\begin{array}{c}\text { Total Number of TBI Cases } \\
\text { Based on Age Range }\end{array}$ & \multicolumn{3}{c}{ Ps14 Prediction Accuracy } & \multicolumn{3}{c}{ PSD Prediction Accuracy } \\
\hline Age (Years) & Age (Years) & \multicolumn{1}{c}{ Identified Correctly } & Misidentified & \multicolumn{2}{c}{ Identified Correctly } & Misidentified \\
\hline $\mathbf{1 7 - 6 5}$ & $\geq \mathbf{6 6}$ & $\mathbf{1 7 - 6 5}$ & $\geq \mathbf{6 6}$ & $\mathbf{1 7 - 6 5}$ & $\mathbf{6 6}$ & $\mathbf{1 7 - 6 5}$ & $\geq \mathbf{6 6}$ & $\mathbf{1 7 - 6 5}$ & $\geq \mathbf{6 6}$ \\
\hline \multirow{2}{*}{26} & 198 & 6 & 83 & 20 & 115 & 21 & 89 & 5 & 109 \\
& $(26.0 \%)$ & $(41.9 \%)$ & $(76.2 \%)$ & $(58.0 \%)$ & $(80.7 \%)$ & $(44.9 \%)$ & $(19.3 \%)$ & $(55.0 \%)$ \\
\hline
\end{tabular}


Table 9 provides an analysis of the relationship between injury parameters (categorized according to Table 5) and the percentage of cases that survived (number 1224 cases) and those that had not survived (number 224 cases) in the validation set. Considering the cases that had not survive, $77.2 \%$ had AIS = 5, 37.1\% were categorized as GCS = severe, 30.8\% categorized as pulse rate as abnormal, $26.8 \%$ were categorized as respiration rate as abnormal and $71.4 \%$ were categorized as systolic blood pressure as abnormal. These results indicate that AIS, GCS and systolic blood pressure to be particularly sensitive indicators for identifying cases that did not survive.

Table 9. Analysis of injury parameters in relation to cases that survived and those that had not survived.

\begin{tabular}{ccccc}
\hline \multirow{2}{*}{ Parameters Injury Grade } & All Subjects & Survived & Did Not Survive \\
\cline { 2 - 5 } & & $\mathbf{1 4 4 8}$ & $\mathbf{1 2 2 4}$ & $\mathbf{2 2 4}$ \\
\hline \multirow{2}{*}{ AIS } & 2 & $12(0.8 \%)$ & $12(1.0 \%)$ & $0(0.0 \%)$ \\
& 3 & $159(11.0 \%)$ & $154(12.6 \%)$ & $5(2.2 \%)$ \\
& 5 & $597(41.2 \%)$ & $551(45.0 \%)$ & $46(20.5 \%)$ \\
\multirow{2}{*}{ GCS (categorized) } & 1 (Severe) & $147(10.2 \%)$ & $64(5.2 \%)$ & $83(37.1 \%)$ \\
& 2 (Moderate) & $133(9.2 \%)$ & $98(8.0 \%)$ & $35(15.6 \%)$ \\
\multirow{2}{*}{ PR (categorized) } & 3 (Mild) & $1168(80.7 \%)$ & $1062(86.8 \%)$ & $106(47.3 \%)$ \\
& 2 (Abnormal) & $338(23.3 \%)$ & $269(22.0 \%)$ & $69(30.8 \%)$ \\
\multirow{2}{*}{ RR (categorized) } & 1 (Abnormal) & $1110(76.7 \%)$ & $955(78.0 \%)$ & $155(69.2 \%)$ \\
\hline \multirow{2}{*}{ SBP (categorized) } & 2 (Normal) & $236(16.3 \%)$ & $176(14.4 \%)$ & $60(26.8 \%)$ \\
& 1 (Abnormal) & $7212(83.7 \%)$ & $1048(85.6 \%)$ & $164(73.2 \%)$ \\
\hline
\end{tabular}

Table 10 provides the mean and standard deviation of AIS and categorized GCS, pulse rate (PR), respiratory rate (RR) and systolic blood pressure (SBP) in cases included in the validation data set that had not survive. The results from this table confirm the conclusion derived from Table 9 with regard to the particular significance of GCS, AIS and SBP. The mean (categorized) systolic blood pressure is close to the abnormal value while the mean GCS is close to moderate severity and AIS represent high injury severity.

Table 10. Provides the mean and standard deviation of AIS and categorized Glasgow comas score (GCS), pulse rate (PR), respiratory rate (RR) and systolic blood pressure (SBP).

\begin{tabular}{ccc}
\hline Parameters & Mean & Standard Deviation \\
\hline AIS & 4.75 & 0.48 \\
GCS (categorized) & 2.10 & 0.92 \\
PR (categorized) & 1.69 & 0.46 \\
RR (categorized) & 1.73 & 0.44 \\
SBP (categorized) & 1.29 & 0.45 \\
\hline
\end{tabular}

Figure 10 shows the interrelationship between AIS and categorized SBP (Figure 10a) and GCS and categorized SBP for cases included in the validation dataset that had not survived. The figure indicates that the great majority of cases with AIS $=5$ had abnormal SBP. The relationship between GCS and systolic blood pressure is not as well defined as that for AIS and systolic blood pressure. 


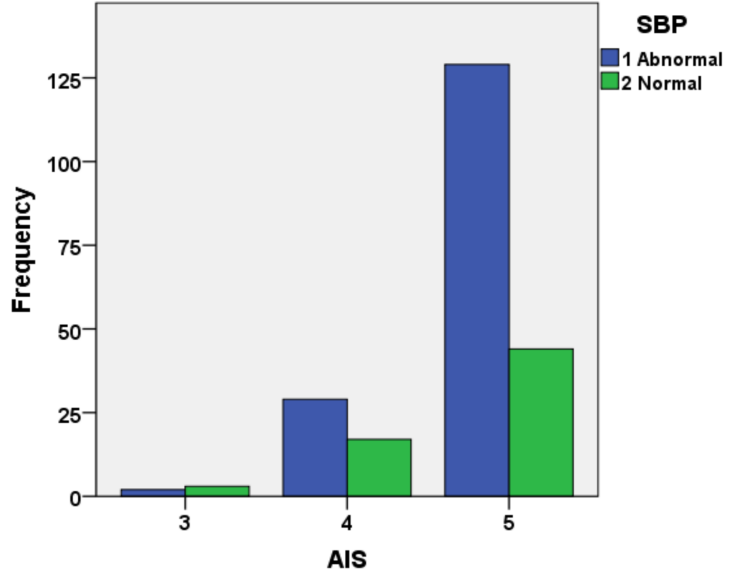

(a)

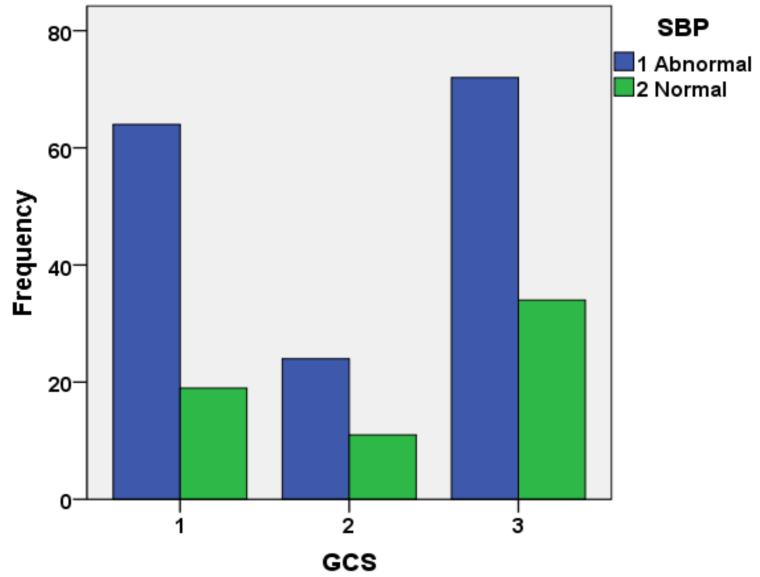

(b)

Figure 10. Relationship between (a) AIS and systolic blood pressure; (b) GCS and systolic blood pressure for cases that were included in the validation set and had not survived. Blue $=$ abnormal category, Green = normal category.

In order to explore the effects of respiration rate, systolic blood pressure and pulse rate on the accuracy of PSD in identifying the cases included in the validation set, each parameter was separately excluded and PSD identification accuracy was determined. The results are summarized in Table 11. The use of GCS and AIS on their own sharply reduced the effectiveness of PSD, resulting in $55.1 \%$ and $31.3 \%$ correct identification of the surviving and not surviving cases. Inclusion of the age with AIS and GCS significantly improved the PSD performance resulting in $82.4 \%$ and $65.2 \%$ correct identification for the surviving and not surviving cases. The inclusion of systolic blood pressure with age, AIS and GCS resulted in $83.3 \%$ and $64.3 \%$ correct identification of surviving and not-surviving cases.

Table 11. Illustration of the effect of age, pulse rate (PR), systolic blood pressure (SBP) and respiratory rate (RR) on PSD performance in identifying surviving and not-surviving cases included in the validation set.

\begin{tabular}{ccccccccc}
\hline \multirow{2}{*}{$\begin{array}{c}\text { Number of Cases in the } \\
\text { Validation Set }\end{array}$} & \multicolumn{2}{c}{$\begin{array}{c}\text { Correct PSD } \\
\text { Identification Using } \\
\text { AIS and GCS Only }\end{array}$} & $\begin{array}{c}\text { Correct PSD Identification } \\
\text { Using AIS and GCS with } \\
\text { Age Only }\end{array}$ & $\begin{array}{c}\text { Correct PSD Identification } \\
\text { Using AIS, GCS, Age } \\
\text { and SBP }\end{array}$ & $\begin{array}{c}\text { Correct PSD Identification } \\
\text { Using AIS, GCS, PR, SBP, } \\
\text { RR and Age }\end{array}$ \\
\hline \multirow{2}{*}{ Survived } & $\begin{array}{c}\text { Did Not } \\
\text { Survive }\end{array}$ & Survived & $\begin{array}{c}\text { Did Not } \\
\text { Survive }\end{array}$ & Survived & $\begin{array}{c}\text { Did Not } \\
\text { Survive }\end{array}$ & Survived & $\begin{array}{c}\text { Did Not } \\
\text { Survive }\end{array}$ & $\begin{array}{c}\text { Survived } \\
\text { Did Not } \\
\text { Survive }\end{array}$ \\
\hline \multirow{2}{*}{1224} & 224 & $\begin{array}{c}675 \\
(55.1 \%)\end{array}$ & $70(31.3 \%)$ & $\begin{array}{c}1008 \\
(82.4 \%)\end{array}$ & $146(65.2 \%)$ & $\begin{array}{c}1019 \\
(83.3 \%)\end{array}$ & $144(64.3 \%)$ & 1112 \\
\hline
\end{tabular}

The study evaluated the performance of PSD in determining the probability of survival in adult subjects with TBI. It highlighted some complexities in determining the probability of survival. An issue is related to the interrelationships of injury parameters and other factors such as age, sex, pre-existing medical conditions that can influence the probability of survival [41]. AIS, GCS, age, respiration rate, pulse rate and systolic blood pressure play an important role in determining the probability of survival in TBI cases. We are currently working on improving the performance of models for injury outcome analysis. In this study PSD was compared with Ps14. The newer version of Ps14, i.e., Ps17, is similar to Ps14 but its coefficients were changed slightly. In this study we did not have access to Ps17 values from the TARN database but in future we will compare its performance with PSD when Ps17 values become available to us.

\section{Conclusions}

A comparison of predictive statistical diagnosis (PSD) and Ps14 for determining the probability of survival in adult subjects with traumatic brain injury (TBI) was carried out. PSD uses Bayesian statistics while Ps14 uses regression. Overall PSD gave an improved performance in identifying those that 
had not survived. However in some injury patterns, Ps14 was more accurate. The interrelationships between the parameters used in the models (Ps14 and PSD) and the manners they affected their identification accuracy were studied. The performance of the two models was different for the younger group (aged 17-65 years) than the older group (aged above 65). The input parameters to PSD, i.e., AIS, GCS, pulse rate, respiration rate, systolic blood pressure and age were found to be important indicators of TBI outcome (surviving or not surviving). The exclusion of any the parameters in the PSD model altered its performance. For example with all 6 parameters included, PSD identified $90.8 \%$ of the surviving and $50.0 \%$ of non-survivors but by excluding pulse rate and respiration rate, PSD identified $83.3 \%$ of the surviving cases and $64.3 \%$ of non-survivors.

The study showed that PSD can be valuable in analyzing TBI outcomes; however, the number of non-survivors included in the study may have not been sufficiently high to indicate the full potential of the PSD and thus a further study with a larger number of cases will be beneficial.

Acknowledgments: We are grateful for the ongoing support Trauma Audit and Research Network (TARN) has provided for the study by allowing access to their trauma injury database and for the very valuable discussions. In particular we are very grateful to: Antoinette Edwards (Executive Director), Maralyn Woodford (Director of Strategy), Gemma Reed (Executive Assistant) and Phil Hammond (Injury Coding Supervisor) for their great assistance.

Author Contributions: All authors jointly conceived and designed the experiments; analyzed the data and wrote the paper. All authors have read and approved the manuscript.

Conflicts of Interest: The authors declare no conflict of interest.

\section{References}

1. Mullins, R.J. A historical perspective of trauma system development in the United States. J. Trauma Acute Care Surg. 1999, 47, S8-S14. [CrossRef]

2. Laytin, A.D.; Dicker, R.A.; Gerdin, M.; Roy, N.; Sarang, B.; Kumar, V.; Juillard, C. Comparing traditional and novel injury scoring systems in a US level-I trauma center: An opportunity for improved injury surveillance in low- and middle-income countries. J. Surg. Res. 2017, 215, 60-66. [CrossRef] [PubMed]

3. Dillon, B.; Wang, W.; Mouamra, O. A comparison study of the injury score models. Eur. J. Trauma 2006, 6, 538-547. [CrossRef]

4. Wisner, D.H. History and current status of trauma scoring system. Arch. Surg. 1992, 127, 111-117. [CrossRef] [PubMed]

5. Kim, Y.J. Injury severity scoring systems: A review of application to practice. Nurs. Crit. Care 2012, 17, 138-150. [CrossRef] [PubMed]

6. Fani-Salek, M.H.; Totten, V.Y.; Terezakis, S.A. Trauma scoring systems explained. Emerg. Med. Australas. 1999, 11, 155-166. [CrossRef]

7. Meredith, W.; Rutledge, R.; Hansen, A.F. Field triage of trauma patients based upon the ability to follow commands. J. Trauma Inj. Infect. Crit. Care 1995, 38, 129-134. [CrossRef]

8. Reith, F.C.M.; Lingsma, H.F.; Gabbe, B.J.; Lecky, F.E.; Roberts, I.; Maas, A.I.R. Differential effects of the Glasgow coma scale score and its components: Analysis of 54,069 patients with traumatic brain injury. Injury 2017, 48, 1932-1943. [CrossRef] [PubMed]

9. Moon, J.; Seo, B.; Jang, J.; Lee, J.; Moon, H. Evaluation of probability of survival using trauma and injury severity score method in severe neurotrauma patients. J. Korean Neurosurg. Soc. 2013, 54, 42-46. [CrossRef] [PubMed]

10. Chawda, M.N.; Hildebrand, F.; Pape, H.C.; Giannoudis, P.V. Predicting outcome after multiple trauma: Which scoring system? Injury 2004, 35, 347-358. [CrossRef]

11. Pike, I.; Khalil, M.; Yanchar, N.L.; Tamim, H.; Nathens, A.B.; Macpherson, A.K. Establishing an injury indicator for severe paediatric injury. Inj. Prev. 2017, 23, 118-123. [CrossRef] [PubMed]

12. Kuwabara, K.; Matsuda, S.; Fushimi, K.; Ishikawa, K.B.; Horiguchi, H.; Fujimori, K. Probability of survival, early critical care process, and resource use in trauma patients. Am. J. Emerg. Med. 2010, 28, 673-681. [CrossRef] [PubMed] 
13. Escobedo, L.V.S.; Habboushe, J.; Kaafarani, H.; Velmahos, G.; Shah, K.; Lee, J. Traumatic brain injury: A case-based review. World J. Emerg. Med. 2013, 4, 252-259. [CrossRef] [PubMed]

14. Menon, D.K.; Schwab, K.; Wright, W.D.; Mass, A.I. Position statement: Definition of traumatic brain injury. Arch. Phys. Med. Rehabil. 2010, 91, 1637-1640. [CrossRef] [PubMed]

15. Chapman, C.J.; Diaz-Arrastia, R. Military traumatic brain injury: A review. Alzheimers Dement. 2014, 10, S97-S104. [CrossRef] [PubMed]

16. Saatchi, R.; Oke, S.; Allen, E.M.; Jervis, B.W.; Hudson, N. Signal processing of the contingent negative variation in schizophrenia using multilayer perceptrons and predictive statistical diagnosis. IEE Proc. Sci. Meas. Technol. 1995, 14, 269-277. [CrossRef]

17. Saatchi, R. Single-trial lambda wave identification using a fuzzy inference system and predictive statistical diagnosis. J. Neural Eng. 2004, 1, 21-31. [CrossRef] [PubMed]

18. Gennarelli, T.A.; Wodzin, E. AIS 2005: A contemporary injury scale. Injury 2006, 37, 1083-1091. [CrossRef] [PubMed]

19. Stevenson, M.; Segui-Gomez, M.; Lescohier, I.; Di Scala, C.; McDonald-Smith, G. An overview of the injury severity score and the new injury severity score. Inj. Prev. 2001, 7, 10-13. [CrossRef] [PubMed]

20. Baker, S.P.; O'Neill, B.; Haddon, W.; Long, W. The injury severity score: A method for describing patients with multiple injuries and evaluating emergency care. J. Trauma 1974, 14, 187-196. [CrossRef] [PubMed]

21. Stoner, H.B.; Barton, R.N.; Little, R.A.; Yates, D.W. Measuring the severity of injury. Br. Med. J. 1977, 2, 1247-1249. [CrossRef] [PubMed]

22. McDonald, S. Impairments in social cognition following severe traumatic brain injury. J. Int. Neuropsychol. Soc. 2013, 19, 1-16. [CrossRef] [PubMed]

23. Middleton, P.M. Practical use of the Glasgow Coma Scale; a comprehensive narrative review of GCS methodology. J. Australas. Emerg. Nurs. 2012, 15, 170-183. [CrossRef] [PubMed]

24. Jennett, B.; Teasdale, G. Aspects of coma after severe head injury. Lancet 1977, 1, 878-881. [CrossRef]

25. Chung, P.; Khan, F. Traumatic brain injury (TBI): Overview of diagnosis and treatment. J. Neurol. Neurolphysiol. 2013, 5, 182-192.

26. Marshall, M.S.; Riechers, R.G. Diagnosis and management of moderate and severe traumatic brain injury sustained in combat. Mil. Med. 2012, 177, 76-85. [CrossRef] [PubMed]

27. Zuercher, M.; Ummenhofer, W.; Baltussen, A.; Walder, B. The use of Glasgow Coma Scale in injury assessment: A critical review. Brain Inj. 2009, 23, 371-384. [CrossRef] [PubMed]

28. Geocadin, R. Traumatic brain injury. In Handbook of Neuro Critical Care; Bhardwaj, A., Mirski, M., Ulatowski, J., Totowa, N.J., Eds.; Humana Press: New York, NY, USA, 2004; pp. 73-89.

29. Prasad, K. The Glasgow Coma Scale: A critical appraisal of its clinimetric properties. J. Clin. Epidemiol. 1996, 49, 755-763. [CrossRef]

30. Champion, H.R.; Sacco, W.J.; Copes, W.S.; Gann, D.S.; Gennarelli, T.A.; Flanagan, M.E. A revision of the trauma score. J. Trauma 1989, 29, 623-629. [CrossRef] [PubMed]

31. Schluter, P.J. The trauma and injury severity score (TRISS) revised. Injury 2011, 42, 90-96. [CrossRef] [PubMed]

32. Siritongtaworn, P.; Opasanon, S. The use of trauma score-injury severity score (TRISS) at Siriraj Hospital: How accurate is it? J. Med. Assoc. Thail. 2009, 92, 1016-1021.

33. Domingues, C.A.; Nogueira, L.S.; Settervall, C.H.S.; Sousa, C. Performance of trauma and injury severity score (TRISS) adjustments: An integrative review. Rev. Esc. Enferm. USP 2015, 49, 138-146. [CrossRef] [PubMed]

34. Trauma Audit and Research Network. Improvements in the Probability of Survival Model. Available online: https: / / www.tarn.ac.uk/Content.aspx?ca $=4 \& c=3515$ (accessed on 5 April 2018).

35. Charlson, M.E.; Charlson, R.E.; Peterson, J.C.; Marinopoulos, S.S.; Briggs, W.M.; Hollenberg, J.P. The Charlson comorbidity index is adapted to predict costs of chronic disease in primary care patients. J. Clin. Epidemiol. 2008, 6, 1234-1240. [CrossRef] [PubMed]

36. Aitchison, J.; Dunsmore, I.R. Statistical Prediction Analysis; Cambridge University Press: Cambridge, UK, 1975.

37. Aitchison, J.; Habbema, J.D.E.; Kay, J.W. A critical comparison of two methods of statistical discrimination. Appl. Stat. 1977, 26, 15-27. [CrossRef] 
38. The Royal Children's Hospital Melbourne. Normal Ranges for Physiological Variables. Available online: https://www.rch.org.au/clinicalguide/guideline_index/Normal_Ranges_for_Physiological_Variables (accessed on 5 April 2018).

39. Moppett, I.K. Traumatic brain injury: Assessment, resuscitation and early management. Br. J. Anaesth. 2007, 99, 18-31. [CrossRef] [PubMed]

40. Janich, K.; Nguyen, H.S.; Patel, M.; Shabani, S.; Montoure, A.; Doan, N. Management of adult traumatic brain injury: A review. J. Trauma Treat. 2016, 5. [CrossRef]

41. Saleh, M.; Saatchi, R.; Lecky, F.; Burke, D. Analysis of the influence of trauma injury factors on the probability of survival. Int. J. Biol. Biomed. Eng. 2017, 11, 88-96.

C 2018 by the authors. Licensee MDPI, Basel, Switzerland. This article is an open access article distributed under the terms and conditions of the Creative Commons Attribution (CC BY) license (http://creativecommons.org/licenses/by/4.0/). 